\section{Controls in studies}

\section{of magnetism}

The appropriateness of controls is often neglected in systematic reviews of complementary therapies. In their systematic review and meta-analysis of static magnets for reducing pain, Max Pittler and colleagues correctly used the appropriateness of a trial's control as a criterion for including the trial in their analysis. ${ }^{1}$

However, for trials that used a weak magnet as their control, Pittler and colleagues failed to specify the maximal strength of the magnetic field that they felt was appropriate for controls, and they did not justify their choice with references to the published literature. They mentioned that it is assumed that magnets must have a strength of $30 \mathrm{mT}$ or greater for therapeutic benefit, but this value was cited from one of the reviewers' own articles and did not appear to be specific to the present study. Such information could be very useful for future studies in this area, especially given the authors' conclusion that "the ideal magnet strength and treatment duration are unclear." Assessing the trials the reviewers had excluded on the basis of the relative strengths of the placebo and test magnets might have had an impact on the outcomes reported.

\section{Peter W. McCarthy PhD}

Welsh Institute of Chiropractic, University of Glamorgan, Pontypridd, UK

Competing interests: None declared.

\section{REFERENCE}

I. Pittler MH, Brown EM, Ernst E. Static magnets for reducing pain: systematic review and meta-analysis of randomized trials. $C M A J 2007 ; 177: 736-42$.

DOI:I0.1503/cmaj.1070158

\section{[One of the authors responds:]}

We welcome Peter McCarthy's comments. As we reported in our article, the primary analysis in our study included only trials that compared magnets and a nonmagnetic placebo. ${ }^{1}$ There was no significant difference in pain reduction between the magnet and placebo groups as measured on a Ioo-mm visual analogue scale, which was defined as the primary outcome. The evidence does not support the use of static magnets for pain relief.

\section{Max H. Pittler MD PhD}

Deputy Director, Complementary

Medicine, Peninsula Medical School,

Universities of Exeter and Plymouth,

Exeter, UK

Competing interests: None declared.

\section{REFERENCE}

I. Pittler MH, Brown EM, Ernst E. Static magnets for reducing pain: systematic review and meta-analysis of randomized trials. $C M A J$ 2007;177:736-42.

DOI:Io.I503/cmaj.1070I63

\section{Combined antithrombotic}

\section{therapy}

The study by Joseph Delaney and colleagues on the use of antithrombotic medications raises many important questions. ${ }^{1}$ In several randomized controlled trials on coronary artery disease, giving oral anticoagulants to patients whose international normalized ratio was maintained between 2 and 3 did not significantly increase the risk of major bleeding. ${ }^{2}$ When added to antiplatelet therapy, oral anticoagulation therapy leads to a marginal but statistically significant reduction of cardiovascular risk. In patients who have atrial fibrillation, deep vein thrombosis or pulmonary embolism as well as coronary artery disease, the addition of acetylsalicylic acid to oral anticoagulation therapy is important because antiplatelet therapy has very little, if any, effect on the recurrence of deep vein thrombosis and pulmonary embolism and on stroke prevention, and warfarin has a minimal effect on the recurrence of coronary events.

The combination of acetylsalicylic acid and clopidogrel in the CURE trial did not lead to excess major bleeding, although there was some minor confounding with the use of glycoprotein IIb-IIIa inhibitors. ${ }^{3}$ In support of the authors' findings, the long-term addition of clopidogrel to acetylsalicylic acid in the CHARISMA trial was not beneficial in the population as a whole and did lead to some increase in bleeding. ${ }^{4}$ In patients with acute coronary syndrome and patients who have received a stent, however, dual antiplatelet therapy is crucial and the length of therapy depends upon the clinical setting and stent type. Trial data suggest that clopidogrel therapy should be limited to patients who would most benefit from it. It does not seem prudent to change this practice on the basis of the authors' findings.

The paper by Delaney and colleagues raises the very important question of the combined use of acetylsalicylic acid with nonsteroidal anti-inflammatory drugs. There appears to limited benefit to this drug combination and it should probably be avoided.

The authors imply that warfarin, acetylsalicylic acid and clopidogrel should not be used together because of the risk of gastrointestinal bleeding. Although their findings are certainly interesting, until further work elucidates the reasons for the increased bleeding risk their data should be interpreted with caution.

\section{David E. Good MD}

Fellow in Cardiovascular Medicine, Ochsner Clinic Foundation, New Orleans, La.

Competing interests: None declared.

\section{REFERENCES}

I. Delaney JA, Opatrny L, Brophy JM, et al. Drug-drug interactions between antithrombotic medications and the risk of gastrointestinal bleeding. CMAJ 2007;177:347-51.

2. Anand SS, Yusuf S. Oral anticoagulants in patients with coronary artery disease. J Am Coll Cardiol 2003;4I(4 Suppl S):62S-69S.

3. Yusuf S, Zhao F, Mehta SR, et al; Clopidogrel in Unstable Angina to Prevent Recurrent Events Tria Investigators. Effects of clopidogrel in addition to aspirin in patients with acute coronary syndromes without ST-segment elevation. $N$ Engl J Med 200I; 345:494-502.

4. Bhatt DL, Fox KAA, Hacke W, et al; CHARISMA Investigators. Clopidogrel and aspirin versus aspirin alone for the prevention of atherothrombotic events. N Engl J Med 2006;354:I706-I7.

DOI:I0.1503/cmaj.I070122

\section{[The authors respond:]}

We read with interest David Good's comments on our paper on drug-drug interactions between antithrombotic 